\title{
Fixed sets and endpoints of set-valued asymptotic contractions in metric spaces
}

\author{
M. Fakhar, Z. Soltani, and J. Zafarani
}




\title{
FIXED SETS AND ENDPOINTS OF SET-VALUED ASYMPTOTIC CONTRACTIONS IN METRIC SPACES
}

\author{
M. FAKHAR, Z. SOLTANI, AND J. ZAFARANI \\ Received 24 September, 2013
}

\begin{abstract}
By introducing a new concept called "set-valued asymptotic contraction of final type" in metric spaces, the existence and uniqueness of compact fixed sets for such mappings have been obtained. Furthermore, by adding additional conditions, we prove the existence and uniqueness of endpoints for these maps.

2010 Mathematics Subject Classification: 47H10; 54C60

Keywords: fixed set, endpoint, asymptotic contraction of the final type, approximate endpoint property, topological contraction
\end{abstract}

\section{INTRODUCTION AND PRELIMINARIES}

In 2003, Kirk [8] introduced the notion of asymptotic contraction on metric spaces and proved a fixed point theorem for such contractions. Later on, Suzuki [11] introduced the notion of asymptotic contraction of Meir-Keeler and extended Kirk's fixed point theorem. Recently, Suzuki [12] introduced the concept of asymptotic contraction of final type for single valued mappings and proved the following result.

Theorem 1 (Suzuki [12]). Let $(X, d)$ be a complete metric space and let $T$ be a continuous mapping on $X$. Assume that $T$ is asymptotic contraction of final type (ACF, for short), i.e., the following contractions hold:

(C1) $\lim _{\delta \rightarrow+0} \sup \left\{\limsup _{n \rightarrow \infty} d\left(T^{n}(x), T^{n}(y)\right): d(x, y)<\delta\right\}=0$.

(C2) For each $\varepsilon>0$, there exists $\delta>0$ such that for $x, y \in X$ with $\varepsilon<d(x, y)<$ $\varepsilon+\delta$, there exists $v \in \mathbb{N}$ such that $d\left(T^{v}(x), T^{v}(y)\right) \leq \varepsilon$.

(C3) For $x, y \in X$ with $x \neq y$, there exists $v \in \mathbb{N}$ such that

$$
d\left(T^{v}(x), T^{v}(y)\right)<d(x, y) .
$$

(C4) For $x \in X$ and $\varepsilon>0$, there exist $\delta>0$ and $v \in \mathbb{N}$ such that

$$
\epsilon<d\left(T^{i}(x), T^{j}(x)\right)<\epsilon+\delta \text { implies } d\left(T^{v} \circ T^{i}(x), T^{v} \circ T^{j}(x)\right) \leq \epsilon
$$

The first author was partially supported by a grant from IPM (No. 92550414) and the Center of Excellence for Mathematics, University of Shahrekord. 
for all $i, j \in \mathbb{N}$.

Then $T$ has a unique fixed point $z \in X$. Moreover, $\lim _{n} T^{n}(x)=z$ holds for every $x \in X$.

Suzuki also mentioned that this result is the final generalization in some senses.

In recent years, many authors have studied the existence and uniqueness of fixed sets and endpoints for set-valued maps in metric spaces and topological spaces; see $[1-7,9,10,13-18]$ and references therein. Recently Fakhar [6] extended the Kirk's asymptotic contraction to set-valued maps and proved the existence and uniqueness of endpoints for those contractions which have the approximate endpoint property. Here, we introduce the notion of set-valued asymptotic contraction of final type in metric spaces and the existence and uniqueness of compact fixed set of these contractions are proved. Then, by using this result, we prove the existence and uniqueness of endpoint for such mappings which are topological contraction or they have the approximate endpoint property.

Let us introduce some definitions and facts which will be used in the sequel. Let $X$ be a nonempty set and $T: X \rightarrow 2^{X}$ be a set-valued map with nonempty values. A subset $A$ of $X$ is said to be a fixed set of $T$, if $T(A)=A$. We denote the set of all fixed set of $T$ with $\operatorname{Fix}(T)$. An element $x \in X$ is said to be an endpoint (or stationary point or strict fixed point) of $T$, if $T(x)=\{x\}$. The set of all endpoints of $T$ is denoted by $\operatorname{End}(T)$. Suppose that $X$ is a topological space, a set-valued map $T: X \rightarrow 2^{X}$ is said to be a topological contraction if for every nonempty compact subset $A$ of $X$ with $T(A)=A, A$ is singleton, i.e., $A$ is an endpoint of $T$. Let $(X, d)$ be a metric space and $C B(X)$ and $K(X)$ denote the family of all nonempty closed and bounded subset of $X$ and the family of all nonempty compact subset of $X$ respectively. Then the Hausdorff metric on $C B(X)$ is given by

$$
H(A, B)=\max \{e(A, B), e(B, A)\}
$$

where

$$
e(A, B)=\sup _{a \in A} d(a, B), e(B, A)=\sup _{b \in B} d(b, A) \quad \text { and } \quad d(a, B)=\inf _{b \in B} d(a, b) .
$$

It is well known that if $(X, d)$ is a complete metric space, then the pair $(K(X), H)$ is a complete generalized metric space. We say that a set-valued map $T$ on a metric space $X$ has the approximate endpoint property if

$$
\inf _{x \in X} \sup _{y \in T x} d(x, y)=0 .
$$

\section{MAIN REsults}

In this section we assume that $(X, d)$ is a metric space. In the first step we introduce the set-valued asymptotic contraction of the final type in metric spaces as follows. 
Definition 1. A set-valued map $T: X \rightarrow C B(X)$ is said to be set-valued asymptotic contraction of final type, if the following conditions hold:

(D1) $\lim _{\delta \rightarrow 0^{+}} \sup \left\{\limsup \sup _{n \rightarrow \infty} H\left(T^{n}(x), T^{n}(y)\right): d(x, y)<\delta\right\}=0$.

(D2) For each $\varepsilon>0$, there exists $\delta>0$ such that for $x, y \in X$ with $d(x, y)<\varepsilon+\delta$, there exists $v \in \mathbb{N}$ such that $H\left(T^{v}(x), T^{v}(y)\right)<\varepsilon$.

(D3) For $x \in X$ and $\varepsilon>0$, there exist $\delta>0$ and $v \in \mathbb{N}$ such that $H\left(T^{i}(x), T^{j}(x)\right)<\varepsilon+\delta$ implies $H\left(T^{v} \circ T^{i}(x), T^{v} \circ T^{j}(x)\right)<\varepsilon$ for all $i, j \in \mathbb{N}$.

It's easy to see that, if $\lim _{n \rightarrow \infty} H\left(T^{n}(x), T^{n}(y)\right)=0$ for every $x, y \in X$ and $\left\{T^{n}(x)\right\}$ is a Cauchy sequence respect to Hausdorff metric, then $T$ is set-valued asymptotic contraction of final type. According to this fact we give the following example of a set-valued asymptotic contraction of final type as follows.

Example 1. Let $X=\mathbb{R}$ and $T: X \rightarrow 2^{X}$ be defined as follows:

$$
T(x)= \begin{cases}\{0\} & x<0 ; \\ {\left[1,2-\frac{x}{2}\right]} & 0 \leq x<1 ; \\ \{3\} & 1 \leq x \leq 2 ; \\ {[3,4]} & x>2 .\end{cases}
$$

Since $T^{n}(x)=[3,4]$ for $x \in X$ and $n \geq 4$. Therefore,

$$
\lim _{n \rightarrow \infty} H\left(T^{n}(x), T^{n}(y)\right)=0
$$

for every $x, y \in X$ and $\left\{T^{n}(x)\right\}$ is a Cauchy sequence respect to Hausdorff metric, then $T$ is asymptotic contraction of final type.

Definition 2 ([6]). A set-valued map $T: X \rightarrow C B(X)$ is said to be a set-valued Kirk's asymptotic contraction, if there exists a continuous function $\varphi$ from $[0, \infty)$ into itself and a sequence $\varphi_{n}$ of functions from $[0, \infty)$ into itself satisfying the following:

(A1) $\varphi(0)=0$.

(A2) $\varphi(r)<r$ for $r \in(0, \infty)$

(A3) $\left\{\varphi_{n}\right\}$ is uniformly convergent to $\varphi$

(A4) $H\left(T^{n}(x), T^{n}(y)\right)<\varphi_{n}(d(x, y))$ for all $n \in \mathbb{N}$ and $x, y \in X$.

In the following result we show that any set-valued Kirk's asymptotic contraction is a set-valued asymptotic contraction of final type.

Proposition 1. Suppose that $T$ is a set-valued Kirk's asymptotic contraction on $X$. Then $T$ is a set-valued asymptotic contraction of final type.

Proof. Similar to Proposition 3 in [11], we put $E=\{d(x, y): x, y \in X\}$ and define a sequence $\left(\psi_{n}\right)$ of function from $[0, \infty)$ into itself by

$$
\psi_{n}(t)=\left\{\begin{array}{cl}
\varphi_{n}(t)+\frac{t}{n} & \text { if } t \in E, \\
0 & \text { if } t \notin E,
\end{array}\right.
$$


when the sequence $\left(\varphi_{n}\right)$ is defined in Definition 2 corresponding to $T$.

Now, we prove the following conditions :

(B1) $\lim \sup _{n} \psi_{n}(\varepsilon) \leq \varepsilon$ for all $\varepsilon \geq 0$,

(B2) For each $\varepsilon>0$, there exist $\delta>0$ and $\nu \in \mathbb{N}$ such that $\psi_{v}(t) \leq \varepsilon$ for all $t \in$ $[0, \varepsilon+\delta]$

(B3) $H\left(T^{n}(x), T^{n}(y)\right)<\psi_{n}(d(x, y))$ for all $n \in \mathbb{N}$ and $x, y \in X$ with $x \neq y$.

It is obvious that $\left(\psi_{n}\right)$ satisfies (B1) and (B3). Let us prove (B2). Fix $\varepsilon>0$. Since $\varphi(\varepsilon)<\varepsilon$ and $\varphi$ is continuous, we can choose $\delta>0$ such that $\delta<\frac{(\varepsilon-\varphi(\varepsilon))}{2}$ and $\varphi(t)-$ $\varphi(\varepsilon)<\frac{(\varepsilon-\varphi(\varepsilon))}{2}$ for $t \in[\varepsilon-\delta, \varepsilon+\delta]$. Also for $t \in(0, \varepsilon-\delta]$, we have $\varphi(t)<t<\varepsilon-\delta$. For such $\delta$, we also choose $v \in \mathbb{N}$ such that

$$
\frac{(\varepsilon+\delta)}{v}<\frac{\delta}{2} \text { and } \sup \left\{\left|\cdot \cdot(\mathrm{t})-{ }^{\prime}(\mathrm{t})\right|: \mathrm{t} \in \mathrm{E}\right\}<\frac{\mathrm{ffi}}{2} .
$$

We fix $t \in(0, \varepsilon+\delta]$. In the case of $t \notin E$, we have $\psi_{\nu}(t)=0<\varepsilon$. For the case $t \in E$ and $t \in(0, \varepsilon-\delta]$, we have

$$
\psi_{v}(t)=\varphi_{v}(t)+\frac{t}{v} \leq \varphi(t)+\frac{\delta}{2}+\frac{\delta}{2} \leq \varepsilon-\delta+\frac{2 \delta}{2}=\varepsilon .
$$

Also, in the case $t \in E$ and $t \in[\varepsilon-\delta, \varepsilon+\delta]$, we have

$$
\begin{aligned}
\psi_{v}(t) & =\varphi_{v}(t)+\frac{t}{v} \\
& \leq \varphi(t)+\frac{\delta}{2}+\frac{\delta}{2} \\
& \leq \varphi(\varepsilon)+\frac{(\varepsilon-\varphi(\varepsilon))}{2}+\frac{2 \delta}{2} \\
& \leq \varphi(\varepsilon)+\frac{2(\varepsilon-\varphi(\varepsilon))}{2}=\varepsilon,
\end{aligned}
$$

which completes our assertion. Now, we prove $T$ fulfills the conditions (D1), (D2) and (D3). Since

$$
\begin{aligned}
\lim _{\delta \rightarrow 0^{+}} \sup \left\{\limsup _{n \rightarrow \infty} H\left(T^{n}(x), T^{n}(y)\right): d(x, y)<\delta\right\} \\
\leq \lim _{\delta \rightarrow 0^{+}} \sup \left\{\limsup _{n \rightarrow \infty} \psi_{n}(t): t<\delta\right\} \\
\quad \leq \lim _{\delta \rightarrow 0^{+}}=0,
\end{aligned}
$$

hence we obtain (D1). Let $\varepsilon>0$, from (B2), there exist $\delta>0$ and $v \in \mathbb{N}$ such that $\psi_{\nu}(t) \leq \varepsilon$ for all $t \in[0, \varepsilon+\delta]$. Then for each $x, y \in X$ with $d(x, y)<\varepsilon+\delta$, from (B3), we have

$$
H\left(T^{v}(x), T^{v}(y)\right)<\psi_{\nu}(d(x, y)) \leq \varepsilon,
$$


which implies (D2).

For (D3), let $x \in X$ such that $H\left(T^{i}(x), T^{j}(x)\right)<\varepsilon+\delta$ for all $i, j \in \mathbb{N}$. So

$$
e\left(T^{i}(x), T^{j}(x)\right)<\varepsilon+\delta
$$

and $e\left(T^{j}(x), T^{i}(x)\right)<\varepsilon+\delta$. By the first inequality, we have

$$
\forall z \in T^{i}(x), \exists w \in T^{j}(x) \text { such that } d(z, w)<\delta+\varepsilon .
$$

Therefore, by (D2), for all $z \in T^{i}(x)$, there exists $v \in \mathbb{N}$ such that

$$
H\left(T^{v}(z), T^{v}(w)\right)<\varepsilon
$$

that deduces $e\left(T^{v}(z), T^{v}(w)\right)<\varepsilon$, so for all $z \in T^{i}(x), e\left(T^{v}(z), T^{v}\left(T^{j}(x)\right)\right)<\varepsilon$. Then, $e\left(T^{v}\left(T^{i}(x)\right), T^{v}\left(T^{j}(x)\right)\right)<\varepsilon$. By the same argument, we have

$$
e\left(T^{v}\left(T^{j}(x)\right), T^{v}\left(T^{i}(x)\right)\right)<\varepsilon .
$$

Therefore,

which implies (D3).

$$
H\left(T^{\mathcal{v}}\left(T^{i}(x)\right), T^{\mathcal{v}}\left(T^{j}(x)\right)\right)<\varepsilon,
$$

Example 1 and Theorem 9 of [12] and Proposition 3 of [11], show that set-valued asymptotic contraction of the final type is strictly weaker than set-valued Kirk's asymptotic contraction.

Now, we give the main result as follows.

Theorem 2. Let $(X, d)$ be a complete metric space. Suppose that $T$ is a setvalued asymptotic contraction of final type. Assume that $T$ is continuous and compact valued. Then $T$ has a unique fixed set $A \in K(X)$. Moreover, $\lim _{n} H\left(T^{n}(B), A\right)=0$ holds for every $B \in K(X)$.

Proof. We consider function $F: K(X) \rightarrow K(X)$ defined by $F(A)=T(A)=$ $\cup_{x \in A} T(x)$ for all $A \in K(X)$. Since $T$ is continuous and compact valued, then $F$ is well defined. Also, the continuity of $T$ implies the continuity of $F$. We will claim that $F$ also fulfills condition (D1), (D2) and (D3). Fix $\varepsilon>0$. From (D1) for $T$, there exists $\delta>0$ such that

$$
\sup \left\{\limsup _{n \rightarrow \infty} H\left(T^{n}(x), T^{n}(y)\right): d(x, y)<\delta\right\}<\varepsilon .
$$

So for each $x, y \in X$ such that $d(x, y)<\delta$, there exists $m_{0}>1$ such that for $n>$ $m_{0}, H\left(T^{n}(x), T^{n}(y)\right)<\varepsilon$. Let $A, B \in K(X)$ such that $H(A, B)<\delta$, we show that $H\left(F^{n}(A), F^{n}(B)\right)<\varepsilon$. If $H(A, B)<\delta$, then $e(A, B)<\delta$ and $e(B, A)<\delta$. So by the first inequality,

$$
\forall z \in A, \exists w \in B \quad \text { such that } d(z, w)<\delta,
$$

It follows that for $n>m_{0}, H\left(T^{n}(z), T^{n}(w)\right)<\varepsilon$. Thus, $e\left(T^{n}(z), T^{n}(w)\right)<\varepsilon$. Therefore, $e\left(T^{n}(z), T^{n}(B)\right)<\varepsilon$ for all $z \in A$. We deduce that

$$
e\left(T^{n}(A), T^{n}(B)\right)<\varepsilon \text {. }
$$


From $e(B, A)<\delta$, by the same argument, we obtain $e\left(T^{n}(B), T^{n}(A)\right)<\varepsilon$. Hence, $H\left(T^{n}(A), T^{n}(B)\right)<\varepsilon$ and so,

$$
\sup \left\{\limsup _{n \rightarrow \infty} H\left(T^{n}(A), T^{n}(B)\right): H(A, B)<\delta\right\}<\varepsilon .
$$

Let $\varepsilon>0$, from condition (D2), there exists $\delta>0$ such that for $x, y \in X$ with $d(x, y)<\varepsilon+\delta$, there exists $v \in \mathbb{N}$ such that $H\left(T^{v}(x), T^{v}(y)\right) \leq \varepsilon$. Let $A, B \in K(X)$ such that $H(A, B)<\delta+\varepsilon$, we show that $H\left(F^{\nu}(A), F^{v}(B)\right)<\varepsilon$. If $H(A, B)<\delta+\varepsilon$, then $e(A, B)<\delta+\varepsilon$ and $e(B, A)<\delta+\varepsilon$. So by the first inequality,

$$
\forall z \in A, \exists w \in B \quad \text { such that } d(z, w)<\delta+\varepsilon .
$$

Hence, there exists $v \in \mathbb{N}$ such that

$$
H\left(T^{v}(z), T^{v}(w)\right)<\varepsilon .
$$

Thus, $e\left(T^{v}(z), T^{v}(w)\right)<\varepsilon$, it follows that, for all $z \in A, e\left(T^{v}(z), T^{v}(B)\right)<\varepsilon$, we deduce that $e\left(T^{v}(A), T^{v}(B)\right)<\varepsilon$. From $e(B, A)<\delta+\varepsilon$, by the same argument, we obtain $e\left(T^{\nu}(B), T^{\nu}(A)\right)<\varepsilon$. Hence, $H\left(T^{\mathcal{v}}(A), T^{\mathcal{v}}(B)\right)<\varepsilon$ and so

$$
H\left(F^{v}(A), F^{v}(B)\right)<\varepsilon,
$$

which shows that $F$ satisfies condition (D2).

Now, from condition (D3), there exists $\delta>0$ and $v \in \mathbb{N}$ such that for $x \in X$ with

$$
H\left(T^{i}(x), T^{j}(x)\right)<\varepsilon+\delta \text { implies } H\left(T^{v} \circ T^{i}(x), T^{v} \circ T^{j}(x)\right) \leq \varepsilon \text { for all } i, j \in \mathbb{N} \text {. }
$$

Let $A \in K(X)$ such that $H\left(F^{i}(A), F^{j}(A)\right)<\varepsilon+\delta$, we will show that

$$
H\left(F^{v} \circ(A), F^{v} \circ(A)\right)<\varepsilon .
$$

Since $H\left(T^{i}(A), T^{j}(A)\right)<\varepsilon+\delta$, then

$$
e\left(T^{i}(A), T^{j}(A)\right)<\varepsilon+\delta
$$

and

$$
e\left(T^{i}(A), T^{j}(A)\right)<\varepsilon+\delta .
$$

So by the first inequality,

$$
\forall z \in T^{i}(A), \exists w \in T^{j}(A) \quad \text { such that } d(z, w)<\delta+\varepsilon .
$$

Hence, from condition (D2), there exists $v \in \mathbb{N}$ such that

$$
H\left(T^{v}(z), T^{v}(w)\right)<\varepsilon .
$$

Thus, $e\left(T^{v}(z), T^{v}(w)\right)<\varepsilon$. It follows that $e\left(T^{v}(z), T^{v}\left(T^{j}(A)\right)\right)<\varepsilon$ for all $z \in$ $T^{i}(A)$, which deduces that $e\left(T^{v}\left(T^{i}(A)\right), T^{v}\left(T^{j}(A)\right)\right)<\varepsilon$. From $e\left(T^{i}(A), T^{j}(A)\right)<$ $\varepsilon+\delta$, by the same argument, we obtain

$$
e\left(T^{v}\left(T^{j}(A)\right), T^{v}\left(T^{i}(A)\right)\right)<\varepsilon .
$$

Hence, $H\left(T^{v}\left(T^{i}(A)\right), T^{v}\left(T^{j}(A)\right)\right)<\varepsilon$ and so

$$
\left.\left.H\left(F^{v} \circ F^{i}(A)\right), F^{\mathcal{v}} \circ F^{j}(A)\right)\right)<\varepsilon,
$$


which completes our claim. Therefore, $F$ is a single-valued asymptotic contraction of final type. Hence, by Theorem $1, F$ has a unique fixed point $A \in K(X)$, i.e., $T(A)=F(A)=A$. Moreover, $\lim _{n} H\left(F^{n}(B), A\right)=0$ holds for every $B \in K(X)$.

As a consequence of Proposition 1 and Theorem 2 we obtain the following result.

Corollary 1. Let $(X, d)$ be a complete metric space and T be a set-valued Kirk's asymptotic contraction on $X$. If $T$ is continuous and compact valued, then $T$ has a compact fixed set.

Theorem 3. Let $(X, d)$ be a complete metric space and $T$ satisfying in the conditions of the above theorem. Suppose that $T$ satisfies one of the following conditions:

(i) $T$ has the approximate endpoint property.

(ii) $T$ is a topological contraction.

Then $T$ has the unique endpoint $x_{0}$.

Proof. By Theorem 2, there is a compact fixed set $A \in K(X)$ such that $T(A)=A$. Let $T$ have the approximate endpoint property. Then

$$
C_{n}=\left\{x \in A: H(x, T(x))=\sup _{y \in T x} d(x, y)\right\} \neq \varnothing,
$$

for each $n \in \mathbb{N}$. It is clear that for each $n \in \mathbb{N}, C_{n} \supseteq C_{n+1}$. Since the map $x \rightarrow$ $H(x, T(x))$ is continuous, we have that $C_{n}$ is closed, so $C_{n}$ is compact. Therefore, $\cap_{n \in \mathbb{N}} C_{n}=C \neq \varnothing$. Thus, for $x \in C, H(x, T(x))=\sup _{y \in T x} d(x, y)=0$, and so $T(x)=\{x\}$. If $T$ is a topological contraction then by definition, $A$ is a singleton that is there exists $x_{0} \in X$ such that $T\left(x_{0}\right)=\left\{x_{0}\right\}=A$. Since $T$ has a unique fixed set $A$ in $K(X)$, then $T$ has a unique endpoint.

By the above theorem and Proposition 1, we have the following result.

Corollary 2. Let $(X, d)$ be a complete metric space and $T$ be a set-valued Kirk's asymptotic contraction. Assume that $T$ is continuous and compact valued. Suppose that $T$ satisfies one of the following conditions:

(i) $T$ has the approximate endpoint property.

(ii) $T$ is a topological contraction.

Then $T$ has the unique endpoint $x_{0}$.

Remark 1. Since continuous set-valued map with compact valued and compact domain is uniformly continuous, the above corollary extends Theorem 2.3 in [6].

\section{ACKNOWLEDGEMENT}

The authors would like to thank the referees for their valuable remarks. 


\section{REFERENCES}

[1] A. Amini-Harandi, "Endpoints of set-valued contractions in metric spaces," Nonlinear Anal., Theory Methods Appl., Ser. A, Theory Methods, vol. 72, no. 1, pp. 132-134, 2010.

[2] J.-P. Aubin and I. Ekeland, Applied nonlinear analysis, ser. Pure and Applied Mathematics. A Wiley-Interscience Publication. New York: John Wiley \& Sons, 1984.

[3] J.-P. Aubin and J. Siegel, "Fixed points and stationary points of dissipative multivalued maps," Proc. Am. Math. Soc., vol. 78, pp. 391-398, 1980.

[4] M. Boriceanu, M. Bota, and A. Petruşel, "Multivalued fractals in $b$-metric spaces," Cent. Eur. J. Math., vol. 8, no. 2, pp. 367-377, 2010.

[5] R. Brooks, K. Schmitt, and B. Warner, "Fixed set theorems for discrete dynamics and nonlinear boundary-value problems," Electron. J. Differ. Equ., vol. 2011, p. 15, 2011.

[6] M. Fakhar, "Endpoints of set-valued asymptotic contractions in metric spaces," Appl. Math. Lett., vol. 24, no. 4, pp. 428-431, 2011.

[7] M. Fakhar, Z. Soltani, and J. Zafarani, "Some asymptotic stationary point theorems in topological spaces," Topology Appl., vol. 159, no. 16, pp. 3453-3460, 2012.

[8] W. A. Kirk, "Fixed points of asymptotic contractions," J. Math. Anal. Appl., vol. 277, no. 2, pp. 645-650, 2003.

[9] E. A. Ok, "Fixed set theory for closed correspondences with applications to self-similarity and games," Nonlinear Anal., Theory Methods Appl., Ser. A, Theory Methods, vol. 56, no. 3, pp. 309_ 330, 2004.

[10] E. A. Ok, "Fixed set theorems of Krasnoselskiǔ type," Proc. Am. Math. Soc., vol. 137, no. 2, pp. 511-518, 2009.

[11] T. Suzuki, "Fixed-point theorem for asymptotic contractions of Meir-Keeler type in complete metric spaces," Nonlinear Anal., Theory Methods Appl., Ser. A, Theory Methods, vol. 64, no. 5, pp. 971-978, 2006.

[12] T. Suzuki, "A definitive result on asymptotic contractions," J. Math. Anal. Appl., vol. 335, no. 1, pp. 707-715, 2007.

[13] E. Tarafdar and X.-Z. Yuan, "Set-valued topological contractions," Appl. Math. Lett., vol. 8, no. 6, pp. 79-81, 1995.

[14] E. Tarafdar and X.-Z. Yuan, "The set-valued dynamic systems and its applications to Pareto optima," Acta Appl. Math., vol. 46, no. 1, pp. 93-106, 1997.

[15] K. Włodarczyk, D. Klim, and R. Plebaniak, "Existence and uniqueness of endpoints of closed setvalued asymptotic contractions in metric spaces," J. Math. Anal. Appl., vol. 328, no. 1, pp. 46-57, 2007.

[16] K. Włodarczyk and R. Plebaniak, "Endpoint theory for set-valued nonlinear asymptotic contractions with respect to generalized pseudodistances in uniform spaces," J. Math. Anal. Appl., vol. 339, no. 1, pp. 344-358, 2008.

[17] K. Włodarczyk, R. Plebaniak, and C. Obczyński, "Endpoints of set-valued dynamical systems of asymptotic contractions of Meir-Keeler type and strict contractions in uniform spaces." Nonlinear Anal., Theory Methods Appl., Ser. A, Theory Methods, vol. 67, no. 6, pp. 1668-1679, 2007.

[18] G. X.-Z. Yuan, KKM theory and applications in nonlinear analysis, ser. Pure and Applied Mathematics. New York, NY: Marcel Dekker, 1999, vol. 218.

Authors' addresses

\section{Fakhar}

Department of Mathematics, University of Isfahan, Isfahan, 81745-163, Iran 
School of Mathematics, Institute for Research in Fundamental Sciences (IPM), P. O. Box : 19395-5746, Tehran, Iran

E-mail address: fakharesci.ui.ac.ir

\section{Z. Soltani}

Department of Mathematics, Faculty of Science, University of Kashan, Kashan, 87317-51167, Iran

E-mail address: z.soltani@kashanu.ac.ir

J. Zafarani

Department of Mathematics, Sheikhbahaee University and University of Isfahan, Isfahan, Iran

E-mail address: jzafezafarani.ir 